\title{
A atuação do(a) psicólogo(a) hospitalar na assistência ao paciente crítico
}

\author{
The role of the hospital psychologist in the care of critical patients \\ El papel del psicólogo del hospital em la atención de pacientes críticos
}

Lucas de Almeida Modesto ${ }^{1 *}$, Tony Anderson Xavier Teles ${ }^{1}$, Hellen Cristiane de Freitas Lima ${ }^{1}$, Icaro Mercides Almeida Tonin ${ }^{1}$, Ana Carolina Araujo de Almeida Lins².

\section{RESUMO}

Objetivo: Verificar qual a atuação do profissional de psicologia na assistência ao paciente crítico através de uma revisão integrativa da literatura, buscando evidenciar o perfil dos trabalhos publicados nas fontes de indexação de maior impacto em âmbito nacional, de modo a possibilitar um melhor direcionamento dos estudos relativos a este conceito. Métodos: Foram utilizadas as bases de dados eletrônicas Scientific Electronic Library Online (SciELO) e Periódicos Eletrônicos de Psicologia (PePSIC). Os critérios de inclusão são: artigos indexados e disponíveis na íntegra no período de janeiro de 2009 a setembro de 2019; que estejam no idioma português; com temáticas que sejam pertinentes ao objetivo dessa revisão. Resultados: A atuação do psicólogo hospitalar com o paciente crítico necessita de técnicas e formas de intervenções que deem conta das especificidades no tratamento deste referente a procedimentos de outros profissionais, além do local em que este paciente pode se encontrar. A atuação neste contexto pode envolver o paciente, a família e a equipe multidisciplinar. Considerações finais: Os estudos investigados apontam que a atuação do psicólogo leva em consideração tanto a vontade do paciente quanto a melhor terapêutica proposta pela equipe, além das múltiplas formas que a família pode auxiliar no tratamento quando orientada corretamente.

Palavras-chave: Psicologia, Hospital, Terapia intensiva.

\section{RESUME}

Objective: To verify the professional performance of psychology in the care of critical patients through an integrative literature review, looking for evidence or profiles of works published in the indexing sources of greatest impact at the national level, in order to allow a better direction of studies related to this concept. Methods: We use Electronic Scientific Library Online (SciELO) and Electronic Psychology Journals (PePSIC) as electronic database. Inclusion requirements are: articles indexed and available in full from January 2009 to September 2019; that are in the Portuguese language; with themes that are relevant to the purpose of this review. Results: a performance of the hospital psychologist with the critical patient, using techniques and forms of application that consider the specificities in the treatment of this reference procedure for other professionals, in addition to the place where this patient can find. Performance in this context can involve the patient, a family and a multidisciplinary team. Final considerations: The investigated studies indicate that the psychologist's performance takes into account both the patient's will and the best therapeutic approach proposed by the team, in addition to the multiple ways that the family can help in the treatment when properly oriented.

Keywords: Psychology, Hospital, Intensive care.

\footnotetext{
${ }^{1}$ Centro Universitário Metropolitano da Amazônia (UNIFAMAZ), Belém - PA.

*E-mail: modestolucas16@gmail.com

2 Universidade Federal do Pará (UFPa), Belém - PA.
} 
RESUMEN

Objetivo: Verificar el desempeño profesional de la psicología en el cuidado de pacientes críticos a través de una revisión bibliográfica integradora, buscando evidencia o perfiles de trabajos publicados en las fuentes de indexación de mayor impacto a nivel nacional, a fin de permitir una mejor dirección de los estudios relacionados con este concepto. Métodos: Usamos Electronic Scientific Library Online (SciELO) y Electronic Psychology Journals (PePSIC) como base de datos electrónica. Los requisitos de inclusión son: artículos indexados y disponibles en su totalidad desde enero de 2009 hasta septiembre de 2019; que están en el idioma portugués; con temas que son relevantes para el propósito de esta revisión. Resultados: un desempeño del psicólogo del hospital con el paciente crítico, utilizando técnicas y formas de aplicación que consideran las especificidades en el tratamiento de este procedimiento de referencia para otros profesionales, además del lugar donde ese paciente puede encontrar. El desempeño en este contexto puede involucrar al paciente, una familia y un equipo multidisciplinario. Consideraciones finales: Los estudios investigados indican que el desempeño del psicólogo tiene en cuenta tanto la voluntad del paciente como el mejor enfoque terapéutico propuesto por el equipo, además de las múltiples formas en que la familia puede ayudar en el tratamiento cuando se orienta adecuadamente.

Palabras clave: Psicología, Hospital, Terapia intensiva.

\section{INTRODUÇÃO}

A inserção da psicologia tendo o hospital como campo de atuação no Brasil se deu por volta da década de 1970. No entanto, a psicologia hospitalar só foi regulamenta como especialidade pelo Conselho Federal de Psicologia (CFP) aproximadamente 30 anos depois, no ano de 2000, através da resolução 14/2000 (COSTA VADSF, et al., 2009).

A implementação desse campo profissional suscitou a utilização de métodos e técnicas de diversas áreas da psicologia, como da psicologia clínica, utilizando também aspectos organizacionais, sociais e educacionais (SCHNEIDER AMB e MOREIRA MC, 2017).

Desse modo, a psicologia hospitalar é um campo da psicologia que pretende humanizar a prática dos profissionais de saúde dentro do ambiente hospitalar. Esta prática redefine os aportes teóricos acerca da somatização e todas as intercorrências que se dão nesse processo de adoecimento.

Embora a psicologia hospitalar tenha emergido no ramo da psicologia clínica, desenvolveu-se e assumiu métodos próprios, adaptando-se a realidade hospitalar para atender as necessidades dos pacientes, familiares e equipe, uma vez que todo o trabalho do(a) psicólogo(a) hospitalar se dá de forma multi e interdisciplinar, o que proporciona constantes trocas de conhecimentos e um atendimento mais integral ao paciente, isto é, olhando para o indivíduo como um todo, na sua dimensão biopsicossocial (VIEIRA MC, 2010).

Para Camon VAA, et al. (1994), a psicologia hospitalar tem o principal objetivo de minimizar o sofrimento provocado pelo processo de hospitalização. Dessa maneira, é necessário abranger não apenas a hospitalização em si, mas também as sequelas e consequências emocionais desse processo.

Portanto, a hospitalização não deve ser entendida como apenas um processo de institucionalização, mas como um conjunto de fatos que decorrem a partir desse processo e suas implicações na vida do sujeito hospitalizado.

A atuação do(a) profissional de psicologia nesse contexto se dá no desenvolvimento de atividades em diferentes níveis de tratamento, que podem ser realizadas na avaliação e acompanhamento das demandas psíquicas dos pacientes que estarão ou que já estão sendo submetidos a procedimentos médicos, com foco na promoção e/ou recuperação da saúde mental e física.

Entre as intervenções possíveis do(a) psicólogo(a) hospitalar estão o olhar atento para as relações médico/paciente, paciente/família, paciente/paciente, bem como do próprio processo de adoecimento e 
hospitalização e todos os percalços emocionais que podem emergir a partir destes (CONSELHO FEDERAL DE PSICOLOGIA, 2007). Segundo o CFP (2007), essa forma de atuação se dá nos níveis secundário e terciário de atenção em saúde, e dentre suas atividades estão: o atendimento psicoterápico, os grupos terapêuticos, os atendimentos em ambulatório e nas Unidades de Terapia Intensiva (UTI's), além da assistência no pronto atendimento, em enfermarias em geral, a avaliação psicológica, o psicodiagnóstico, e etc.

Dessa forma, sabe-se que muitos dos pacientes que estão nesses ambientes hospitalares se encontram em estado crítico. No que tange ao paciente crítico, apesar de um vasto acervo de artigos nacionais trabalharem esta temática, nesta pesquisa houve a necessidade de buscar em produções internacionais conteúdos que pudessem conceituar de forma objetiva quem seria esse paciente crítico.

Diante disto, nesta revisão integrativa de literatura (RIL) adotaremos o conceito de paciente crítico descrito por Consuegra RVG (1998) que apresenta este sujeito como o paciente com risco iminente de morte, cujas funções vitais estão gravemente alteradas.

A literatura nacional aponta geralmente o paciente crítico como o sujeito que se encontra na unidade de terapia intensiva. Todavia Zandomenighi RC, et al. (2014) salientam que em pacientes de qualquer unidade de internação oferecida pelos serviços de saúde podem ocorrer complicações que os tornem pacientes críticos.

Por isso, estes pacientes podem se encontrar no Serviço Hospitalar de Emergência (SHE), no Serviço Integrado de Atendimento ao Trauma (SIATE) ou pelo Serviço Móvel de Urgência (SAMU). Os autores também apontam que estes pacientes podem permanecer nas suas respectivas unidades de internação por tempo indeterminado, devido geralmente o número de leitos das Unidades de Terapia Intensiva (UTI's) serem insuficientes.

No que se refere à assistência do(a) profissional de psicologia junto ao paciente crítico, a literatura nacional aponta apenas para o contexto das UTI's, onde Schneider AMB e Moreira MC (2017) descrevem que a assistência psicológica junto a esses pacientes deve considerar os fatores emocionais relativos ao processo de hospitalização, o adoecimento e as práticas invasivas que o paciente crítico está ou estará submetido.

Ademais, este(a) profissional deve verificar se o paciente neste contexto tem a compreensão acerca de seu diagnóstico, motivo da internação em regime hospitalar. Além disso, os autores apontam ainda que essa atuação da psicologia se estende à família e aos visitantes do paciente neste estado crítico, explicando-Ihes as rotinas da UTI e permitindo-Ihes expressar seus sentimentos diante do processo de internalização do seu ente querido.

Por conseguinte, este estudo visa responder a seguinte questão norteadora: Qual é a atuação do(a) psicólogo(a) hospitalar na assistência ao paciente crítico? Entretanto, indo para além do contexto da UTI, verificando se há diferenças no tratamento deste paciente devido sua condição de saúde e muitas vezes a terapêutica necessita que pode requerer que o profissional de psicologia dinamize a sua forma de atuação para oferecer um atendimento psicológico eficaz para o indivíduo que está com risco iminente de morte.

Além disso, a depender o contexto pode influenciar em como este profissional vai prestar sua escuta de forma qualificada. Diante de essas pontuações elencar a necessidade de uma formação qualificada a este profissional.

\section{MÉTODOS}

O presente estudo trata-se de uma revisão integrativa da literatura. O material foi acessado por meio das bases eletrônicas e de acesso aberto Scientific Electronic Library Online (SciELO) e Periódicos Eletrônicos de Psicologia (PePSIC). Os descritores utilizados foram: "psicologia hospitalar" e "paciente crítico". Destacase que foi utilizado "AND" e "OR" entre os descritores. Além de utilizar o filtro Brasil/língua portuguesa para otimizar os artigos ao idioma que se pretende trabalhar. Os critérios de inclusão e exclusão ocorreram conforme a Figura 1. 
Figura 1 - Fluxograma referente aos critérios de inclusão e exclusão.

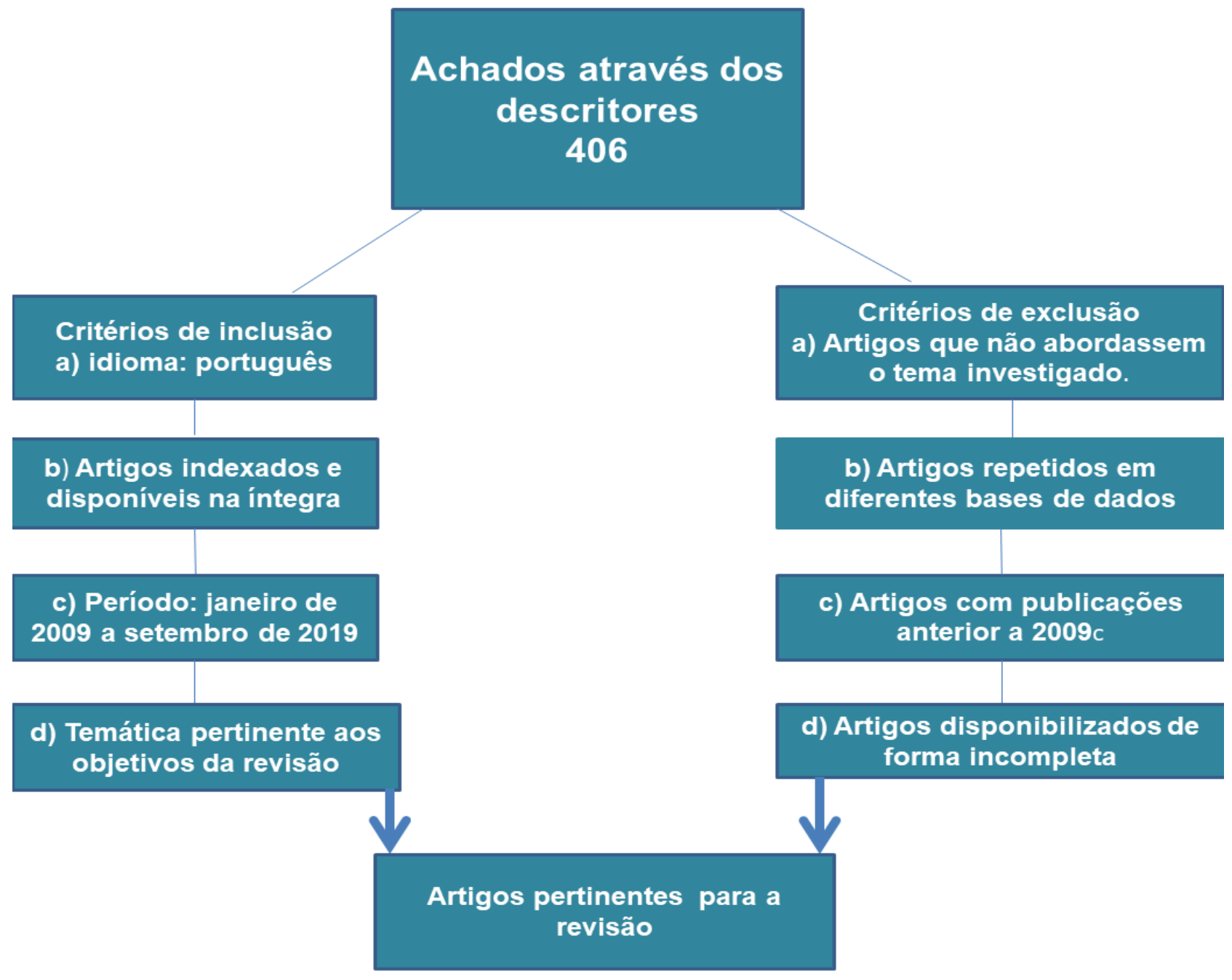

Fonte: Modesto LA, et al.,2019.

O início da pesquisa se deu através da elaboração da pergunta norteadora, que é: qual a atuação do(a) psicólogo(a) hospitalar na assistência ao paciente crítico? A partir de então se realizou a busca inicial, que ocorreu no período de setembro a novembro de 2019.

O levantamento do material deu-se por meio da leitura dos resumos dos artigos, que foram verificados no sentido de considerar se as produções em questão atenderam aos critérios previamente estabelecidos. Elaborou-se então um instrumento para a coleta das informações, composto pelos seguintes itens: procedência, título do artigo, autores, periódico (vol., №, pág., ano), considerações/temática.

As análises e a categorização dos dados coletados procederam em sínteses de estudos por definição do campo de análise da pesquisa/estudo a partir da subdivisão em: título, objetivo, metodologia, atuação do psicólogo e paciente crítico.

\section{RESULTADOS}

Foram encontrados nas bases de dados um total de 406 artigos, quando se utilizou "OR" entre os descritores, visto que quando se utilizou "AND" não apareceram resultados, o que denota que ainda não há uma relação direta na literatura nacional das temáticas. Na leitura dos resumos foram pré-selecionados 31 artigos, contudo ao aplicarem-se os critérios de inclusão e exclusão na leitura integra pode-se trabalhar com 16 artigos, que contemplam a temática de forma direta ou indireta, conforme o Quadro 1. 
Quadro 1 - Análises e categorização dos dados coletados.

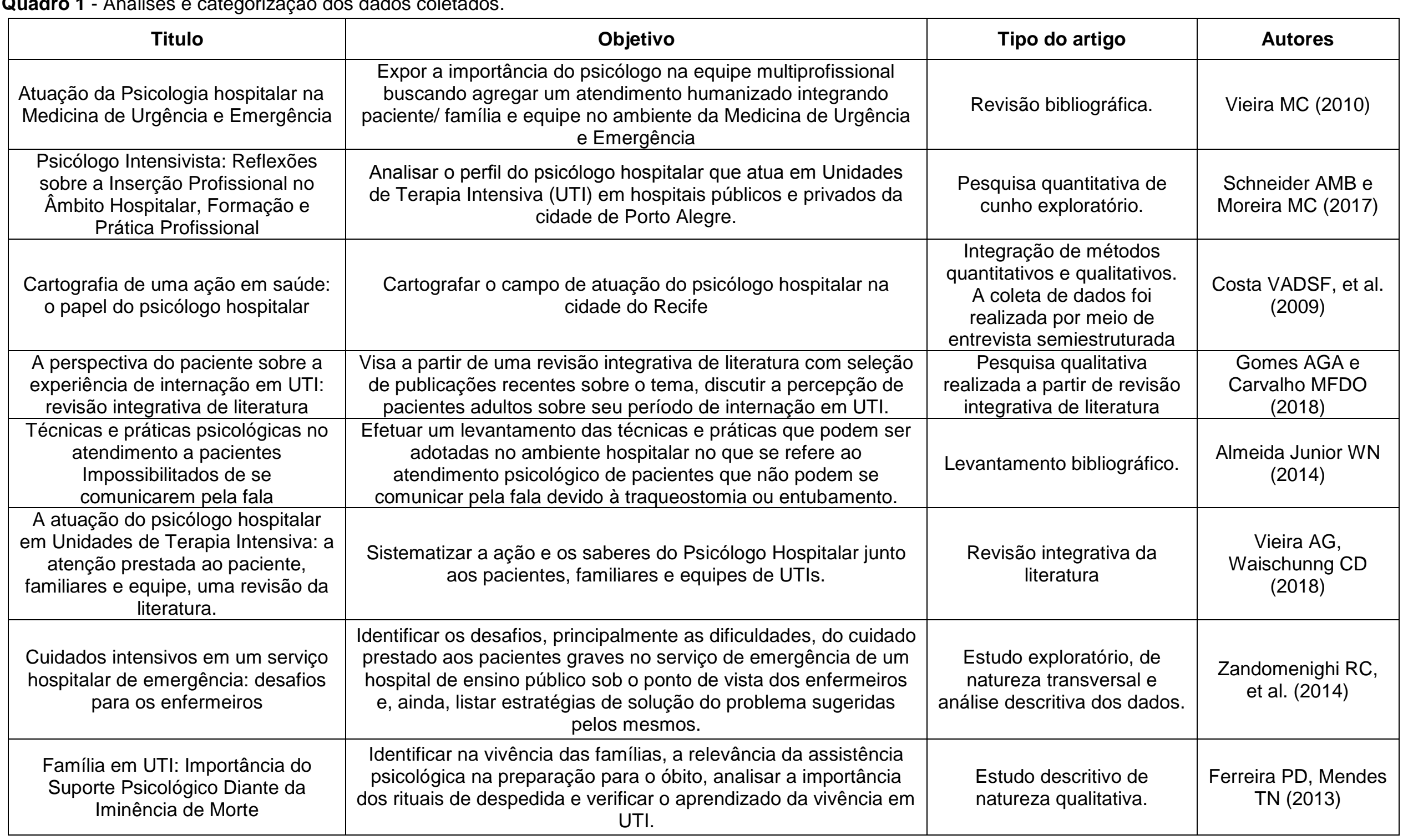

REAS/EJCH | Vol.12(10) | e3649 | DOI: https://doi.org/10.25248/reas.e3649.2020 Página $\mathbf{5}$ de 10 


\begin{tabular}{|c|c|c|c|}
\hline $\begin{array}{l}\text { Avaliação da dor em pacientes } \\
\text { críticos por meio da Escala } \\
\text { Comportamental de Dor. }\end{array}$ & $\begin{array}{c}\text { Descrever e caracterizar a dor e o uso de analgesia no serviço } \\
\text { de urgência e cuidados intensivos. }\end{array}$ & $\begin{array}{l}\text { Estudo transversal com } \\
\text { abordagem quantitativa. }\end{array}$ & $\begin{array}{l}\text { Oliveira LS, et al. } \\
\qquad(2019)\end{array}$ \\
\hline $\begin{array}{l}\text { Enfermeiros recém-formados e o } \\
\text { cuidado intensivo em unidades de } \\
\text { pacientes não-críticos. }\end{array}$ & $\begin{array}{l}\text { Identificar as representações sociais de enfermeiros recém- } \\
\text { formados sobre o cuidado intensivo de Enfermagem ao paciente } \\
\text { crítico hospitalizado em unidades de pacientes não-críticos }\end{array}$ & $\begin{array}{l}\text { Pesquisa de campo, } \\
\text { qualitativa e descritiva. }\end{array}$ & $\begin{array}{l}\text { Almeida RDO, et al. } \\
\qquad(2019)\end{array}$ \\
\hline A prática da psicologia da saúde & Entender o campo da Psicologia Aplicada à saúde & $\begin{array}{l}\text { Levantamento bibliográfico, } \\
\text { considerando a Psicologia } \\
\text { da Saúde no Brasil e em } \\
\text { outros países. }\end{array}$ & $\begin{array}{l}\text { Almeida RAD e } \\
\text { Malagris LEN (2011) }\end{array}$ \\
\hline $\begin{array}{l}\text { Representação social da Psicologia } \\
\text { Hospitalar para familiares de } \\
\text { pacientes hospitalizados em } \\
\text { Unidades de Terapia Intensiva } \\
\end{array}$ & $\begin{array}{c}\text { Identificar a representação social da Psicologia Hospitalar para } \\
\text { os familiares de pacientes hospitalizados em Unidade de Terapia } \\
\text { Intensiva (UTI) e minimizar o sofrimento em relação à } \\
\text { hospitalização }\end{array}$ & $\begin{array}{l}\text { Pesquisa de campo } \\
\text { qualitativa e descritiva. }\end{array}$ & $\begin{array}{l}\text { Moreira EKCB, et al } \\
\qquad(2012)\end{array}$ \\
\hline $\begin{array}{l}\text { Psicologia da Saúde crítica no } \\
\text { contexto hospitalar }\end{array}$ & $\begin{array}{l}\text { Apresentar o desenvolvimento histórico da psicologia da saúde, } \\
\text { mostrando o surgimento das perspectivas tradicionais e crítica e } \\
\text { a partir dessa psicologia da saúde crítica, apresentar algumas } \\
\text { questões para o trabalho do psicólogo no contexto hospitalar. }\end{array}$ & Revisão da literatura. & $\begin{array}{l}\text { Carvalho DBD } \\
\quad(2013)\end{array}$ \\
\hline $\begin{array}{l}\text { A importância da assistência } \\
\text { psicológica junto ao paciente em } \\
\text { hemodiálise }\end{array}$ & $\begin{array}{l}\text { Refletir sobre a atuação do psicólogo junto às pacientes } \\
\text { portadores de insuficiência renal crônica (IRC) em tratamento } \\
\text { hemodialítico; Elucidar a importância do psicólogo como membro } \\
\text { da equipe de nefrologia na assistência ao paciente desde o } \\
\text { diagnóstico à indicação ao tratamento. }\end{array}$ & Revisão bibliográfica. & $\begin{array}{l}\text { Pascoal M, et al. } \\
\qquad(2009)\end{array}$ \\
\hline $\begin{array}{c}\text { Atuação do psicólogo nos hospitais } \\
\text { da Grande Vitória/ES: uma } \\
\text { descrição }\end{array}$ & $\begin{array}{l}\text { Verificar a existência de profissionais de psicologia nos hospitais } \\
\text { de região metropolitana da Grande Vitória/ES; Descrever as } \\
\text { atividades por eles desenvolvidas e seus objetivos de trabalho; } \\
\text { Discutir a inserção destes profissionais nos hospitais e as } \\
\text { principais dificuldades por eles encontradas. }\end{array}$ & Pesquisa de campo. & Avellar LZ (2011) \\
\hline $\begin{array}{l}\text { Adoecimento e finitude: } \\
\text { considerações sobre a abordagem } \\
\text { interdisciplinar no Centro de } \\
\text { Tratamento Intensivo Oncológico }\end{array}$ & $\begin{array}{c}\text { Trazer à tona considerações sobre o trabalho interdisciplinar no } \\
\text { acolhimento à família dos pacientes do Centro de Tratamento } \\
\text { Intensivo adulto do Hospital do Câncer I, enquanto proposta no } \\
\text { que tange ao atendimento de necessidades de suporte social, } \\
\text { clínico e psicológica. }\end{array}$ & $\begin{array}{l}\text { Relato de experiência no } \\
\text { atendimento aos } \\
\text { familiares/responsáveis dos } \\
\text { pacientes internados no CTI } \\
\text { adulto. }\end{array}$ & $\begin{array}{l}\text { Alcantara LDS, et al } \\
\qquad(2013)\end{array}$ \\
\hline
\end{tabular}

Fonte: Modesto LA, et al, 2019.

REAS/EJCH | Vol.12(10) | e3649 | DOI: https://doi.org/10.25248/reas.e3649.2020 Página 6 de 10 
Houve maior concentração de publicações de artigos no ano de 2013: 3 (três) artigos. Entretanto, não houve diferença discrepante nos demais anos do recorte, sendo eles 2009, 2011, 2014, 2018 e 2019 em que foram publicados 2 (dois) artigos respectivamente. Nos anos de 2010, 2012, 2016 e 2017 apenas 01 (uma) publicação foi feita.

No que tange a metodologia dos artigos analisados, foram elas: 5 (cinco) revisões bibliográficas, 4 (quatro) revisões da literatura, 5 (cinco) pesquisas de campo, 2 (dois) estudos transversais e 1 (um) relato de experiência. Com relação aos conceitos que abarcam a temática dessa pesquisa, 12 (doze) artigos trouxeram discussões relativas tanto ao paciente crítico, como a atuação do psicólogo hospitalar; 3 (três) artigos trataram apenas da atuação do (a) psicólogo (a) no contexto hospitalar, e 2 (dois) trataram especificamente sobre paciente crítico.

\section{DISCUSSÃO}

A despeito de o termo paciente crítico ser relativamente novo, não consta na literatura analisada uma conceituação exata para o que seria esse paciente. Geralmente ele é remetido ao individuo que se encontra internado nas Unidades de Terapia Intensiva (AVELLAR LZ, 2011); (FERREIRA PD e MENDES TN, 2013); (GOMES AGA e CARVALHO MFDO, 2018); (VIEIRA AG e WAISCHUNNG CD, 2018).

Todavia, outros autores apontam que esse paciente também está em outros setores de internação, como Oliveira LS, et al. (2013) que apontam que este paciente, além da UTI, está nos Serviços de Urgência e Emergência. Segundo Moreira EKCB, et al. (2012), alguns pacientes críticos encontram-se nos leitos das enfermarias, nos corredores dos hospitais, nos centros cirúrgicos. Este é o paciente internado, seja por uma pneumonia, diabetes, infarto, etc., ou que veio dos pós-cirúrgicos, seja devido a um acidente de carro, a um câncer, etc.

Corroborando com esta ideia, Almeida RDO, et al. (2019) descreve o paciente crítico como aquele que precisa de atendimento intensivo, não necessariamente estando em uma UTI, mas podendo estar nas unidades clínicas, cirúrgicas, centros diagnósticos, emergências, unidades básicas de saúde, domicílios ou que tenham sido trazidos pelo SAMU.

Concomitante a presença desse paciente em diversos setores hospitalares, vale mostrar quais as características deste. Segundo Vieira MC (2010), o paciente crítico é aquele que passa por uma mudança intensa de rotina por causa de um mal-estar inusitado ou um acidente por causas diversas, onde as consequências são intercortadas por múltiplos sentimentos e pensamentos. A autora também ressalta que a vida desse sujeito normalmente é desestruturada a partir desse abrupto processo mórbido, seja por um acidente ou por uma condição médica complicada.

Diante disto, pode-se também considerar pacientes críticos como pacientes de hemodiálise, por exemplo, que Pascoal M, et al. (2009) descrevem como sujeitos que têm uma mudança brutal de rotina, por vezes perdendo seus empregos, com restrições alimentares e apresentando aspectos emocionais variados diante da dependência de um procedimento para permanecerem vivos, tais como: desespero, insegurança, impulsividade, etc.

Outro exemplo de pacientes que podem ser considerados críticos por se encaixarem nessa caracterização são os pacientes oncológicos, devido ao fato de algumas vezes necessitarem de um cuidado intensivo e por terem alterações abruptas em sua rotina. Porém, com o tratamento adequado, podem deixar de serconsiderados pacientes críticos devido a sua melhora clínica (ALCÂNTARA LDS, et al., 2013).

Outras características oriundas desta análise é que o paciente crítico é aquele que tem um risco iminente de morte (FERREIRA PD e MENDES TN, 2013); (VIEIRA AG e WAISCHUNNG CD, 2018), por vezes estão com rebaixamento de nível de consciência, em ventilação mecânica ou fazendo uso de sedativos (OLIVEIRA LS, et al., 2013). Entretanto, este estudo não se refere ao paciente em estágio de terminalidade.

Logo, o manejo do(a) profissional de Psicologia em relação a este paciente tem suas peculiaridades. Contudo, apesar da diversidade de características e locais onde se encontram os pacientes críticos, os artigos analisados não divergem em relação a atuação do(a) profissional da psicologia a estes. 
Uma das premissas principais, e presente na ideia de vários autores, é que a atuação do(a) profissional de psicologia no contexto hospitalar, junto ao paciente crítico, ocorre em uma tríade envolvendo paciente, família e equipe de saúde (VIEIRA MC, 2010); (ALMEIDA RAD E MALAGRIS LEN, 2011); (MOREIRA EKCB et al., 2012); (FERREIRA PD e MENDES TN, 2013);

Schneider AMB e Moreira MC (2017); Gomes AGA e Carvalho MFDO (2018); Vieira AG e Waischunng CD (2018) corroboram a esta ideia apontando a importância desta tríade para uma atuação eficaz do profissional de psicologia, junto ao paciente, a família e a equipe de saúde. .

Em linhas gerais, cabe a esse(a) profissional da psicologia, a promoção do bem-estar, seja individual, coletivo ou relacional (CARVALHO DBD, 2013), além de fomentar a criação de vínculos e a interação da equipe multidisciplinar entre si, com os pacientes e com a família (PASCOAL M,et al., 2009).

No tocante ao paciente, a atuação do(a) psicólogo(a) deve ser focal, breve, entretanto, ressaltando a subjetividade do sujeito e devendo ser adaptada ao contexto hospitalar (COSTA VADSF, et al., 2009); (VIEIRA MC, 2010); (ALMEIDA RAD e MALAGRIS LEN, 2011). Além disso, deve ainda proporcionar mecanismos de enfrentamento diante do processo de adoecimento, facilitando a implicação do paciente ao tratamento, trabalhando as questões desfavoráveis e adaptativas, além de ser o(a) porta-voz dos desejos e necessidades deste paciente (VIEIRA MC, 2010). Em síntese, o(a) profissional de psicologia deve minimizar o sofrimento decorrente do processo de adoecimento e internação (SCHNEIDER AMB e MOREIRA MC, 2017).

De acordo com Ferreira PD e Mendes TN (2013), o(a) profissional de psicologia deve "marcar presença" (termo utilizados pelos autores) ao lado do paciente, facilitando a expressão de seus sentimentos e emoções e, no contexto da UTI, deve tentar minimizar a solidão que pode ser vivenciada neste contexto. Outro fator preponderante que também abrange a atuação do(a) psicólogo(a) é a função de auxiliar o paciente no processo de adaptação a esse novo momento, bem como à rotina da internação, aos procedimentos invasivos, aos estressores ambientais e a lidar com os sentimentos negativos que possam vir a surgir no ambiente onde possa estar o paciente crítico (GOMES AGA e CARVALHO MFDO, 2018). Doravante a isso, há a necessidade de resgatar a importância da dignidade no sofrimento e o respeito a individualidade da pessoa humana, considerando sua história de vida (VIEIRA AG e WAISCHUNNG CD, 2018).

Vale ressaltar ainda que, há casos em que o paciente crítico tem certas limitações, como dificuldade para verbalizar, seja por rebaixamento do nível de consciência, devido à ventilação mecânica ou a traqueostomia (ALMEIDA JUNIOR WN, 2014); (OLIVEIRA LS, et al., 2019). Contudo, o(a) profissional da psicologia deve lançar mão de técnicas adaptativas para prezar que as necessidades desse paciente sejam atendidas, desde garantindo maior dosagem de analgésicos para a dor que possa estar sentindo e não pode verbalizar (OLIVEIRA LS, et al., 2019), até auxiliando na expressão de suas vontades em relação ao seu tratamento (ALMEIDA JUNIOR WL, 2014).

A literatura também aponta uma necessidade da atuação desse(a) profissional da psicologia diante do risco iminente de morte do paciente. Segundo Gomes AGA e Carvalho MFDO (2018), a postura do(a) profissional de psicologia diante deste risco é a de levar o paciente a relembrar e relatar suas principais experiências na vida, os projetos e vínculos estabelecidos, além de suas representações de morte, prestando um atendimento humanizado, fornecendo estratégias de enfrentamento diante da situação em que se encontra aquele sujeito hospitalizado. Os autores também apontam que este processo gera um significativo desgaste emocional na família do paciente, salientando a necessidade desse(a) profissional acolher, escutar e dar suporte emocional a esses familiares.

Em relação à atuação do(a) psicólogo(a) com a família do paciente crítico, Vieira MC (2010) e Moreira EKCB, et al. (2012) apontam que no processo de internação, a família também deve receber orientações e suporte emocional, Schneider AMB e Moreira MC (2017) corroboram a essa ideia e acrescenta que a família deve ser auxiliada sobre a melhor forma de lidar com as situações estressantes decorrentes desse processo.

Ferreira PD e Mendes TN (2013) descrevem que o(a) psicólogo(a) deve estimular o contato entre os familiares e os pacientes, ainda que apenas nos horários de visita, observando e avaliando o conteúdo verbal e/ou comportamental,e sua influência no quadro clínico. Além disso, os autores também ressaltam que este

REAS/EJCH | Vol.12(10) | e3649 | DOI: https://doi.org/10.25248/reas.e3649.2020 Página 8 de 10 
profissional deve fomentar as interações, conversas, toques e expressões de sentimento entre o familiar/acompanhante e o paciente em estado crítico. Dentre as muitas formas de atuação do(a) profissional da psicologia com a família do paciente, têm-se: o acompanhamento dos familiares através de entrevistas, de oficinas, de grupos terapêuticos, de grupos de apoio com cuidadores, etc. (AVELLAR LZ, 2011).

Além disso, esses profissionais podem ainda auxiliar no manejo das relações sociais, melhorando a comunicação entre equipe e família, amenizando conflitos e diminuídos situações estressoras (GOMES AGA e CARVALHO MFDO, 2018).

Por fim, cabe elencar a atuação do(a) psicólogo(a) com a equipe de saúde que atende o paciente em estado crítico. É importante pontuar que o trabalho de saúde com o paciente crítico é multi e interdisciplinar (VIEIRA MC, 2010); (SCHNEIDER AMB E MOREIRA MC, 2017); (MOREIRA EKCB, et al., 2012); (PASCOAL $M$, et al., 2009), o que pode proporcionar constantes trocas de conhecimento e discussões conjuntas sobre o melhor atendimento ao paciente crítico. Noutra via, o papel do(a) psicólogo(a) é de sensibilizar a equipe sobre os aspectos psicossociais que interferem na comunicação com o paciente e em sua implicação no tratamento (VIEIRA MC, 2010).

A atuação do(a) profissional de psicologia também deve mostrar, de forma geral, como a equipe pode contribuir para a diminuição dos estressores, possíveis geradores de sofrimento ao paciente. Para tanto, estes profissionais devem ser estimulados a ter uma postura receptiva e eficiente (GOMES AGA e CARVALHO MFDO, 2018).

Ferreira PD e Mendes TN (2013) contribuem com essa ideia e acrescentam ainda que o(a) psicólogo(a) deve prestar assistência a equipe, resgatando a tranquilidade e a sensibilidade de cuidar ao próximo, além de proporcionar escuta e orientações importantes a este contexto. Outro aspecto importante apresentado por Gomes AGA e Carvalho MFDO (2018) é que, em caso de perdas, o(a) profissional de psicologia deve assistir a equipe quanto a sentimentos de perda e frustração oriundos desse momento difícil.

\section{CONCLUSÃO}

Apesar de muitos artigos tratarem da atuação do psicólogo hospitalar com o paciente critico e nas diversas áreas da saúde, observou-se que não se tem ainda um conceito fechado sobre o que o categorizaria. No entanto, muitas pesquisas analisadas apontaram o paciente critico como sinônimo de paciente da/na UTI.

Contudo, conforme a integração dos achados dessa revisão de literatura, verificou-se que o paciente critico pode circular em diversos setores da instituição hospitalar, desde o SAMU, SHE, oncológico, hemodiálise, sejam aqueles decorrentes de complicações na clinica medica e até propriamente a UTI, apontando assim a necessidade do(a) psicólogo(a) lançar mão de técnicas diversas para atender esse paciente tendo em vista que muitas vezes ele necessitará passar por terapêuticas que influenciarão no sua comunicação, por exemplo, cabendo ao profissional de psicologia viabilizar a conciliação das vontades do paciente com as terapêuticas propostas pela equipe, além da própria presença da família que pode auxiliar neste processo.

Além disso, conforme consta na literatura, a atuação desse profissional não se dá de forma isolada, devendo levar sempre em consideração a assistência às famílias e a equipe de saúde que também é atravessada pelo processo de adoecimento e risco iminente de morte que este paciente vem enfrentando.

\section{REFERÊNCIAS}

1. ALCANTARA LDS, et al. Adoecimento e finitude: considerações sobre a abordagem interdisciplinar no Centro de Tratamento Intensivo oncológico. Ciência \& Saúde Coletiva, 2013: 2507-2514.

2. ALMEIDA JUNIOR WN. Técnicas e práticas psicológicas no atendimento a pacientes impossibilitados de se comunicarem pela fala. Psicologia Hospitalar, 2014; 24-44.

3. ALMEIDA RAD, MALAGRIS LEN. A prática da psicologia da saúde. Rev. SBPH, 2011;14(2):183-202.

4. ALMEIDA RDO, et al. Enfermeiros recém-formados e o cuidado intensivo em unidades de pacientes não-críticos. Revista Brasileira de Enfermagem, 2019: 254-262. 
5. AVELLAR LZ. Atuação do psicólogo nos hospitais da grande vitória/es: uma descrição. Psicologia em Estudo, 2011; 16(3): 491-499.

6. CAMON VAA, et al. Psicologia Hospitalar: Teoria e Prática. São Paulo: Pioneira Thomson Learning, 1994; 116p.

7. CARVALHO DBD. Psicologia da saúde crítica no contexto hospitalar. Psicologia: Ciência e Profissão, 2013; 33(2).

8. CONSELHO FEDERAL DE PSICOLOGIA. 2007.Resolução CFP N N 013/2007. Brasília. Disponivel em: https://site.cfp.org.br/wp-content/uploads/2008/08/Resolucao_CFP_nx_013-2007.pdf. 2017. p. 32. Acesso em: 28 de set de 2019.

9. CONSUEGRA RVG. Cuidado de enfermeira al paciente crítico. Avances em enfermeira, 1998; 14(2):27-29.

10. COSTA VADSF, et al. Cartografia de uma ação em saúde: o papel do psicólogo hospitalar. SBPH, 2009 12(1): 113134.

11. FERREIRA PD, MENDES TN. Família em UTI: Importância do Suporte Psicológico Diante da Iminência de Morte. Rev. SBPH, 2013; 16(1): 88-112.

12. GOMES AGA, CARVALHO MFDO. A perspectiva do paciente sobre a experiência de internação em UTI: revisão integrativa de literatura. Rev. SBPH, 2018; 21(2): 167-185.

13. MOREIRA EKCB, et al. Representação social da Psicologia Hospitalar para familiares de pacientes hospitalizados em Unidade de Terapia Intensiva. Rev. SBPH, 2012; 15(1): 134-162.

14. OLIVEIRA, LS, et al. Avaliação da dor em pacientes críticos por meio da Escala Comportamental de Dor. BrJP, 2019: 112-116.

15. PASCOAL M, et al. A importância da assistência psicológica junto ao paciente com hemodiálise. Rev. SBPH, 2009; 12: 2-11.

16. SCHNEIDER AMB, MOREIRA MC. Psicólogo Intensivista: Reflexões sobre a inserção profissional no âmbito hospitalar, formação e prática profissional. Trends in Psychology / Temas em Psicologia, 2017: 1225-1239.

17. SOUZA MT, et al. Revisão Integrativa de Literatura: o que é e como fazer. Einstein, 2010; 8: 102-106.

18. VIEIRA AG, WAISCHUNNG CD. A atuação do psicólogo hospitalar em Unidades de Terapia Intensiva: a atenção prestada ao paciente, familiares e equipe, uma revisão da literatura. Rev. SBPH, 2018;21(1): 132-153.

19. VIEIRA MC. Atuação da Psicologia hospitalar na Medicina de Urgência e Emergência. Rev Bras Clin Med, 2010: 513519.

20. ZANDOMENIGHI RC, et al. Cuidados intensivos em um serviço hospitalar de emergência: desafios para os enfermeiros. REME - Rev Min Enferm, 2014: 404-414. 